\title{
Erratum
}

\section{Prevention of Murine Breast Cancer by Vaccination with Tumor Cells Modified by Cytokine-Producing Recombinant Vaccinia Viruses}

Gary R. Peplinski, MD, Kangla Tsung, PhD, Jennifer B. Meko, MD, and Jeffrey A. Norton, MD

[Originally published in Annals of Surgical Oncology 3(1):15-23.]

When this article was published, the heading "Best Basic Science Research Paper" was inadvertently omitted, although this information was included in a footnote on the opening page. Lippincott-Raven regrets the error. 